\title{
Promovendo Habilidades de Solução de Problemas Interpessoais em Crianças
}

\author{
Luciana Carla dos Santos Elias \\ Faculdade de Filosofia, Ciências e Letras de Ribeirão Preto da Universidade de São Paulo \\ Edna Maria Marturano \\ Faculdade de Medicina de Ribeirão Preto da Universidade de São Paulo
}

\begin{abstract}
RESUMO
Habilidades de solução de problemas interpessoais - HSPI são importantes para a adaptação psicossocial dos indivíduos. Este trabalho objetivou avaliar a efetividade de um programa para desenvolvimento de HSPI, aplicado por professoras. Participaram 203 alunos do ensino fundamental, com idade entre seis e dez anos, e suas professoras. As crianças foram alocadas em dois grupos: intervenção e comparação. Os grupos foram comparados quanto às habilidades sociais, HSPI, problemas de comportamento e desempenho acadêmico, antes e depois da intervenção, com os instrumentos Sistema de Avaliação de Habilidades Sociais, Child Interpersonal Problem Solving Test e Teste de Desempenho Escolar. Apenas o grupo de intervenção apresentou melhoras significativas em todas as variáveis investigadas. Destaca-se a importância de programas preventivos na escola.
\end{abstract}

Palavras-chave: Habilidades de solução de problemas interpessoais; problemas de comportamento; desempenho acadêmico.

\begin{abstract}
Fostering interpersonal problem solving skills in children

Interpersonal problem solving skills - IPSS are important for psychosocial adaptation of people. This study aimed to evaluate the effectiveness of a program for the development of HSPI, applied by teachers. Participants were 203 students from a public elementary school, aged between six and ten years, and their teachers. The children were allocated in two groups - intervention and comparison. The groups were evaluated before and after intervention as their social skills, IPSS, behavior problems and academic performance, by means of the Social Skills Rating Scale, Child Interpersonal Problem Solving Test and Academic Performance Test. Only children in the intervention group showed significant improvements in all parameters investigated. These results highlights the importance of prevention programs in the schools.
\end{abstract}

Keywords: interpersonal problem solving skills; behavior problems; academic performance.

A escola se constitui em uma instância legítima de educação para a cidadania, atitudes práticas de não violência e promoção do desenvolvimento. Entretanto, por integrar pessoas e interesses distintos, tem se tornado um ambiente de ocorrência de conflitos interpessoais (Loos \& Zeller, 2007). O desenvolvimento de uma criança que vive sob situação de risco para problemas comportamentais em uma escola que não colabora para a promoção de habilidades pode ter consequências negativas para si e seus pares (Conduct Problems Prevention Research Group, 2010).

No que tange a problemas de comportamento, não há uma definição de consenso na literatura (BolsoniSilva \& Del Prette, 2003). Assim como Bolsoni-Silva,
Paiva e Barbosa (2009), adotamos uma definição funcional na qual os problemas de comportamento dificultam o acesso da criança à aquisição de repertórios relevantes de aprendizagem. Pacheco, Alvarenga, Reppold, Piccinini e Hutz (2005) sugerem que é possível identificar dois grandes grupos de problemas de comportamento: os externalizantes (impulsividade, agressão, agitação, características desafiantes e anti-sociais) e os internalizantes (retraimento, depressão, ansiedade e queixas somáticas). Ambas as classes de comportamento podem ser associadas aos conceitos de excesso e déficit comportamental. Os dois grupos de problemas de comportamento dificultam o desenvolvimento psicossocial da criança (Bolsoni-Silva et al., 2009). 
Comportamentos problemáticos frequentemente estão associados a baixo desempenho escolar, entendido como desempenho inferior ao nível esperado para a idade, habilidade e potencial de um indivíduo (Barreto, Freitas, \& Del Prette, 2011; D'Abreu \& Marturano, 2010; Heydenberk \& Heydenberk, 2007). Crianças com problemas de comportamento também podem apresentar déficit em habilidades sociais (Feitosa, Del Prette, Del Prette, \& Loureiro, 2011). Desse modo, dificuldades adaptativas precoces, expressas em problemas emocionais e/ou comportamentais no contexto escolar, têm sido associadas a trajetórias desfavoráveis (Morgan, Farkas, \& Wu, 2009; Silver, Measelle, Armstrong, \& Essex, 2010).

No desenvolvimento influências transacionais se estabelecem entre os domínios comportamental, acadêmico e interpessoal, em processos de retroalimentação (Burt \& Roisman, 2010; Grimm, Steele, Mashburn, Burchinal, \& Pianta, 2010; Van Lier \& Koot, 2010). Estudos de prevenção (Conduct Problems Prevention Research Group, 2010; Witvliet, Van Lier, Cuijpers, \& Koot, 2009) sinalizam que a chave para o manejo dessa condição de tríplice vulnerabilidade parece ser a vertente interpessoal. A capacidade de se dar bem com pessoas e estabelecer relações interpessoais mutuamente satisfatórias, genericamente conhecida como competência social, sobressai como condição protetora do desenvolvimento.

Sabe-se que a entrada na escola constitui um marco no desenvolvimento do indivíduo visto que amplia suas relações sociais e exige dele novas habilidades essenciais para o posterior sucesso acadêmico e social. Diante desse contexto, cada vez mais encontramos estudos focados nesta fase do desenvolvimento, relacionados a programas de intervenção que buscam melhores competências sociais e emocionais (De Salvo, Mazzarotto, \& Lohr, 2005; McCabe \& Altamura, 2011). A experiência escolar é central para as aquisições sociais e educacionais das crianças em nossa cultura. O enfrentamento de demandas interpessoais requer que elas desenvolvam um repertório cada vez mais elaborado de habilidades sociais, com consequente melhora da qualidade de vida, adaptação social e rendimento acadêmico (Del Prette \& Del Prette, 2003; Feitosa et al., 2011). Dessa maneira, o contexto escolar se configura em um lócus onde as habilidades sociais se desenvolvem.

Caballo (2010) refere-se às habilidades sociais como um conjunto de comportamentos emitidos por um indi- víduo num contexto interpessoal que expressa os sentimentos, atitudes, desejos, opiniões ou direitos desse indivíduo de maneira adequada. Tais habilidades são expressas em comportamentos necessários a uma relação interpessoal bem-sucedida conforme características de cada cultura. As habilidades sociais são aprendidas nos contextos de desenvolvimento, desde os primeiros anos de vida à medida que emergem as diferentes habilidades cognitivas, interações com o ambiente e relações sociais. Del Prette e Del Prette (2005) propõem um sistema de classes interdependentes e complementares de habilidades, que seriam prioritárias no desenvolvimento interpessoal das crianças como: autocontrole e expressividade emocional, civilidade, empatia, assertividade, fazer amizades, solução de problemas interpessoais e habilidades sociais acadêmicas.

As habilidades de solução de problemas interpessoais (HSPI) vêm sendo objeto de estudo de diferentes pesquisadores, como habilidades que têm grande influência no nível de ajustamento e bem-estar apresentado pelo indivíduo. Segundo Shure e Spivack (1982), as HSPI são constituídas de seis capacidades: (1) geração de soluções alternativas: (2) consideração de consequências de atos; (3) desenvolvimento de pensamento meio-fim; (4) desenvolvimento do pensamento social-causal; (5) sensibilidade para problemas; (6) orientação. Segundo os autores, as HSPI definem como o indivíduo pensa a respeito de problemas interpessoais, especificamente como os avalia e enfrenta.

Del Prette e Del Prette (2005) destacam que estudos têm mostrado que as HSPI estão associadas a uma maior capacidade da criança para lidar com as fontes potenciais de estresse, a uma melhora da competência social na adolescência e à diminuição da impulsividade. Para Heydenberk e Heydenberk (2007) as HSPI estão relacionadas a um maior senso de auto-eficácia, afetando as opções de atividades das crianças, seus objetivos, comportamentos e prevendo resultados tão diversos como melhor desempenho acadêmico, competência social e tolerância à frustração. Estudos realizados em nosso meio, envolvendo avaliação e intervenção nas HSPI, em diferentes contextos, apontam para associações e correlações entre HSPI, dificuldades acadêmicas e problemas de comportamento, sugerindo que trabalhos preventivos para o desenvolvimento de HSPI podem atuar como fator de proteção ao desenvolvimento infantil (ver revisão em Elias, Marturano, \& Motta-Oliveira, 2012). 
A aquisição de habilidades sociais na infância pode ser vista com uma das formas diretas de impedir ou controlar o comportamento agressivo (Barker et al., 2010) e, indiretamente, um meio de prevenir o risco de rejeição por pares, ao qual as crianças agressivas estão fortemente expostas (Sturaro, Van Lier, Cuijpers, \& Koot, 2011). Estudos sugerem que o desenvolvimento de habilidades sociais na infância pode se constituir em um fator de proteção (Barker et al., 2010; Rodrigues, Dias, \& Freitas, 2010), estabelecido à medida que o comportamento socialmente habilidoso favorece a obtenção de reforçadores sociais importantes (Gonçalves \& Murta, 2008). Observa-se, ainda, uma co-ocorrência entre habilidades sociais e um amplo conjunto de comportamentos adaptativos, como bom desempenho acadêmico, estratégias de enfrentamento, autocuidado, independência e cooperação (Bandeira, Del Prette, Del Prette, \& Magalhães, 2009; Del Prette \& Del Prette, 2005).

A proposição de programas de habilidades sociais na escola foi destacada por Gresham (2004) ao defender três vertentes de diferentes amplitudes, complementares e sequenciadas, na implantação desses programas na escola: os programas universais, para todas as crianças da escola; os seletivos, em pequenos grupos, para crianças de risco que não se beneficiaram dos universais; e os individualizados, para atendimento das crianças que não respondem positivamente às duas estratégias anteriores.

Um programa de intervenção do tipo universal almejando aumentar habilidades de solução de problemas interpessoais em crianças de ensino fundamental foi implantado e avaliado por Borges e Marturano (2002). Participaram 55 crianças da primeira série, designadas seja para a condição de intervenção ou para a condição de comparação. A intervenção foi adaptada do programa Eu Posso Resolver Problemas - EPRP, de Shure (2006). Os resultados indicaram índices de HSPI superiores entre as crianças da condição intervenção em comparação às crianças da condição controle. As observações feitas ao longo do programa evidenciaram que os alunos que inicialmente se envolviam mais em conflitos diminuíram sua participação nesses eventos durante a intervenção.

$\mathrm{O}$ aperfeiçoamento de programas visando o desenvolvimento de HSPI no próprio ambiente escolar aponta para possibilidades concretas de um trabalho preventivo, já que estimula a competência social, a construção de relacionamentos positivos e condutas so- cialmente mais ajustadas (De Salvo et al., 2005). A primeira autora deste artigo, a partir de experiência com o programa EPRP (Shure, 2006), elaborou e testou um programa de caráter universal para o desenvolvimento das HSPI em ambientes coletivos. O programa, denominado "Posso Pensar", contempla algumas demandas que no EPRP não são de todo atendidas: linguagem e conteúdos adaptados culturalmente; linguagem e atividades menos diretivas, apontando diferentes possibilidades de se explorar um mesmo tema, o que daria um maior suporte ao aplicador do programa; temas que abordam valores humanos, como amizade, colaboração, generosidade, honestidade; menor número de lições de forma a viabilizar a aplicação, mas sem prejuízo de conteúdos, utilizando-se reforços como alternativa. A par com essas peculiaridades, o programa tem em comum com o EPRP, além do ensino sistemático de HSPI, o trabalho com reconhecimento de pistas sociais, reconhecimento das emoções em si e nos outros, autoregulação emocional e empatia.

Diante desse contexto o presente estudo teve por objetivos: (a) avaliar a efetividade do programa Posso Pensar, aplicado em sala de aula pelo professor, quanto a efeitos de aumento das HSPI, aumento das habilidades sociais, redução dos problemas de comportamento e melhora no desempenho acadêmico dos alunos; (b) verificar a manutenção dos resultados obtidos ao término do programa, em avaliação de follow-up (seguimento).

\section{MÉTODO}

\section{Delineamento}

O estudo se efetivou em quatro etapas: avaliação pré-intervenção e alocação dos participantes no grupo de intervenção (GI) ou no grupo de comparação (GC); aplicação do programa no GI; reavaliação de todos os participantes logo após a intervenção no GI e espera no GC; e avaliação de seguimento dos participantes do GI, quatro a seis meses após o término do programa.

\section{Participantes}

Participaram do estudo 203 alunos matriculados em uma escola da rede municipal de educação em cidade do interior paulista, com idade entre seis e dez anos (M $=110$ meses, $\mathrm{DP}=16,69)$. O GI, com 45 meninos e 50 meninas $(M=106$ meses, $D P=11,64)$, foi formado pelos alunos de duas turmas de terceiro ano e uma turma de quinto ano, cujas professoras concordaram em avaliar as crianças nos três momentos e administrar o 
programa mediante treino prévio e supervisão. $\mathrm{O} G \mathrm{G}$, com 55 meninos e 53 meninas $(\mathrm{M}=114$ meses, $\mathrm{DP}=$ 18,80), foi formado com alunos de uma turma de segundo ano, uma de quarto ano e uma de quinto ano, cujas professoras não se dispuseram a aplicar o programa, mas concordaram em avaliar seus alunos.

\section{Instrumentos}

Foram utilizados os seguintes instrumentos:

Child Interpersonal Problem Solving (CHIPS) (Shure \& Spivack, 1985). Avalia as HSPI em crianças de nove a 12 anos, por meio de três histórias de problemas interpessoais, apresentadas oralmente, envolvendo crianças de mesmos sexo e faixa etária do examinando. Em face de cada história, a tarefa da criança é encontrar diferentes soluções, no máximo dez, para o problema enfrentado pelo personagem principal. Os critérios para julgamento e pontuação das respostas são fornecidos no manual do procedimento;

Sistema de Avaliação de Habilidades Sociais, versão brasileira (SSRS-BR). O formulário para professores é composto de três escalas: habilidades sociais (inclui as subescalas responsabilidade/cooperação, asserção, autocontrole, autodefesa e cooperação com os pares), problemas de comportamento (inclui as subescalas de comportamentos externalizantes e internalizantes) e competência acadêmica. No estudo de validação brasileira, o instrumento apresentou índices satisfatórios de consistência interna e estabilidade temporal (Bandeira et al., 2009); e

Teste de Desempenho Escolar (TDE) (Stein, 1994). Avalia as capacidades fundamentais para o desenvolvimento escolar, especificamente escrita, aritmética e leitura, de crianças de primeira a sexta séries, por meio de ditado de palavras, operações matemáticas e leitura de palavras. O manual do teste fornece normas para a classificação do desempenho da criança em relação à idade e à série escolar em que ela se encontra.

\section{Procedimento}

As avaliações pré-intervenção com o CHIPS e o TDE foram realizadas em fevereiro, em espaço cedido pela direção da escola, durante o horário de aula, em dias previamente agendados. As crianças foram avaliadas individualmente por três psicólogas treinadas. As professoras responderam ao SRSS enquanto as demais avaliações ocorriam e somente após o término das avaliações iniciaram a aplicação do programa. No final do ano anterior e início do ano de execução do programa as professoras passaram por aulas teórico-vivenciais sobre habilidades sociais e HSPI. Durante a aplicação do programa a seus alunos receberam supervisão conjunta, na qual se trabalhavam as dificuldades encontradas no processo, como, por exemplo, dificuldades operacionais frente ao ritmo da sala e conflitos entre crianças durante as atividades. Na supervisão também era fornecido o material necessário para o desenvolvimento das atividades. Foram realizados com as professoras 24 encontros, com duração de duas horas e meia, compreendendo as aulas teórico -vivenciais e a supervisão para a aplicação do programa.

O programa Posso Pensar é composto de 40 lições temáticas e interativas que desenvolvem as HSPI por meio de histórias e atividades lúdicas. As lições apresentam complexidade crescente, iniciando no reconhecimento de sentimentos e chegando à escolha da melhor solução para o problema ocorrido, verificando suas consequências. As professoras do GI aplicaram esse programa duas a três vezes por semana em sala de aula, com duração entre 15 e 30 minutos, introduzindo, no dia seguinte ao da aplicação, uma atividade de reforço que não ultrapassava 15 minutos.

Após a conclusão do programa, no final de junho e início de julho, crianças e professoras receberam um certificado de participação e as crianças dos dois grupos foram reavaliadas. No final do mês de novembro e primeira quinzena de dezembro, foram realizadas as avaliações de seguimento no GI.

A análise estatística comparou os dados de GI e GC em cada avaliação, assim como as avaliações sucessivas em cada grupo. As comparações foram feitas por meio de contrastes ortogonais com o modelo linear de efeitos mistos (aleatórios e fixos), utilizado quando as respostas de um mesmo individuo estão agrupadas e a suposição de independência entre as observações num mesmo grupo não é adequada (Schall, 1991). Foi atendido o requisito de distribuição normal dos resíduos com média zero e variância constante. $\mathrm{O}$ ajuste do modelo foi feito por meio do procedimento PROC MIXED, do software SAS ${ }^{\circledR}$ 9.1. Utilizou-se um nível alfa de 0,05 .

O projeto foi aprovado pelo Comitê de Ética da Faculdade de Filosofia, Ciências e Letras de Ribeirão Preto (USP), processo 580/2011-2011.1.1426.59.4, em 24/4/2010, e se desenvolveu com autorização da Secretaria de Educação do município. Pais/responsáveis e professoras assinaram o Termo de 
Consentimento Livre e Esclarecido destinado a cada um. Às crianças do grupo de comparação foi oferecido o treinamento em HSPI após a conclusão do estudo, no contraturno do horário escolar.

\section{RESULTADOS}

A Tabela 1 mostra as estatísticas de GI em HSPI, obtidas por meio do CHIPS, e desempenho escolar, obtidas pelo TDE, nas três avaliações. Para as HSPI, houve aumento significativo nas médias, tanto no número total de soluções, quanto no número de soluções rele- vantes, entre as avaliações pré e pós-intervenção. Já entre a avaliação pós-intervenção e o seguimento houve redução das médias. Uma análise da estimativa da diferença entre as médias das avaliações pré e de seguimento não acusou variação significativa.

No TDE houve aumento significativo entre as avaliações pré e pós-intervenção e as avaliações pós e de seguimento, em todas as medidas: escrita, leitura, aritmética e escore total. Esses resultados indicam que as crianças melhoraram após a intervenção e continuaram a progredir nos meses subsequentes.

Tabela 1

Estatisticas do Grupo de Intervenção em HSPI e Desempenho Escolar, em Três Avaliações

\begin{tabular}{lccccccc}
\hline \multicolumn{1}{c}{ Variável } & $\begin{array}{c}\text { Pré- } \\
\text { Intervenção } \\
\boldsymbol{M}(\boldsymbol{D P})\end{array}$ & $\begin{array}{c}\text { Pós-Intervenção } \\
\boldsymbol{M}(\boldsymbol{D P})\end{array}$ & $\begin{array}{c}\text { Seguimento } \\
\boldsymbol{M}(\boldsymbol{D P})\end{array}$ & $\boldsymbol{t}_{\text {pré-pós }}$ & $\boldsymbol{p}_{\text {pré-pós }}$ & $\boldsymbol{t}_{\text {pós-seg }}$ & $\boldsymbol{p}_{\text {pós-seg }}$ \\
\hline Total soluções CHIPS & $8,99(4,07)$ & $10,35(3,2)$ & $9,38(2,44)$ & $-4,05$ & $<0,0001^{* *}$ & 2,70 & $0,0073^{* *}$ \\
Soluções relevantes CHIPS & $8,58(3,99)$ & $10,04(3,08)$ & $9,07(2,44)$ & $-4,35$ & $<0,0001^{* *}$ & 2,82 & $0,0052^{* *}$ \\
Escrita TDE & $18,45(8,54)$ & $21,14(8,67)$ & $23,29(7,81)$ & $-6,3$ & $<0,0001^{* *}$ & $-4,57$ & $<0,0001^{* *}$ \\
Leitura TDE & $49,23(20,53)$ & $58,96(17,00)$ & $63,22(9,47)$ & $-7,26$ & $<0,0001^{* *}$ & $-2,58$ & $0,0103^{*}$ \\
Aritmética TDE & $10,94(563)$ & $13,43(4,80)$ & $14,44(4,64)$ & $-6,55$ & $<0,0001^{* *}$ & $-2,12$ & $0,0345^{*}$ \\
Escore total TDE & $78,73(31,79)$ & $93,53(2743)$ & $100,95(18,7)$ & $-8,92$ & $<0,0001^{* *}$ & $-3,78$ & $0,0002^{* *}$ \\
\hline
\end{tabular}

Nota: Pré-intervenção: $n=95$; pós-intervenção: $n=91$; seguimento: $n=86$. ${ }^{*} p<0,05$; ${ }^{*} p<0,01$.

A Tabela 2 traz os resultados de GC em HSPI, obtidos por meio do CHIPS, e desempenho escolar, obtidos pelo TDE, em dois momentos de avaliação. Houve redução significativa na média de soluções relevantes entre as avaliações pré e pós-espera. Quanto ao desempenho acadêmico, ocorreu aumento significativo apenas na média de escrita.

Foram realizadas comparações entre GI e GC. Com relação às HSPI, na avaliação inicial GI apresentava média superior a GC quanto ao total de respostas $(t=-$ $2,05, \mathrm{p}<0,0001)$ e de respostas relevantes $(t=-2,04$ e $\mathrm{p}<0,0001)$. Essa diferença se acentuou na avaliação pós-intervenção, para o total $(t=-3,90, \quad \mathrm{p}<0,0001) \mathrm{e}$ para as respostas relevantes $(t=-4,37, \mathrm{p}<0,0001)$. Quanto ao desempenho acadêmico, GI e GC no início não apresentaram diferença. Já na avaliação pós-intervenção GI apresentou médias maiores que $\mathrm{GC}$ em todos os itens avaliados: Escrita $(t=-3,29, \mathrm{p}=0,01)$, Leitura $(t=-8,30, \mathrm{p}=0,006)$, Aritmética $(t=-2,17$,

Tabela 2

Estatísticas do Grupo de Comparação em HSPI e Desempenho Escolar, em Duas Avaliações

\begin{tabular}{lcccc}
\hline \multicolumn{1}{c}{ Variável } & Pré-espera $\boldsymbol{M ( D P )}$ & $\begin{array}{c}\text { Pós-espera } \\
\boldsymbol{M}(\boldsymbol{D P})\end{array}$ & $\boldsymbol{t}_{\text {pré-pós }}$ & $\boldsymbol{p}_{\text {pré-pós }}$ \\
\hline Total soluções CHIPS & $6,90(3,03)$ & $6,43(2,75)$ & 1,31 & 0,191 \\
Soluções relevantes CHIPS & $6,53(2,93)$ & $5,70(2,75)$ & 2,53 & $0,0118^{*}$ \\
Escrita TDE & $16,53(10,01)$ & $18,03(10,02)$ & $-3,23$ & $0,0014^{* *}$ \\
Leitura TDE & $50,26(24,04)$ & $51,51(23,96)$ & $-0,31$ & 0,7577 \\
Aritmética TDE & $11,59(6,71)$ & $11,84(6,44)$ & 1,19 & 0,2348 \\
Escore total TDE & $78,37(38,03)$ & $81,52(37,92)$ & $-1,06$ & 0,2906 \\
\hline
\end{tabular}

Nota: Pré-intervenção: $n=108$; pós-intervenção: $n=105 .{ }^{*} p<0,05$; ${ }^{* *} p<0,01$. 
$\mathrm{p}=0,006)$ e Escore total $(t=-13,48, \mathrm{p}=0,004)$.

A Tabela 3 apresenta as estatísticas de GI em relação às escalas de competência acadêmica, habilidades sociais e problemas de comportamento, obtidas por meio do SSRS-BR, nas três avaliações. Os resultados sugerem ganhos generalizados depois da intervenção, indicados por aumento nas medidas de competência acadêmica e habilidades sociais, a par com redução em todas as medidas de comportamentos problema. Não foram observadas diferenças entre as médias pós-intervenção e de seguimento, sinalizando manutenção dos

Tabela 3

Estatísticas do Grupo de Intervenção em Competência Acadêmica, Habilidades Sociais e Problemas de Comportamento, em Três Avaliações

\begin{tabular}{|c|c|c|c|c|c|c|c|}
\hline Variável & $\begin{array}{c}\text { Pré- } \\
\text { Intervenção } \\
M(D P)\end{array}$ & Pós-Intervenção $M(D P)$ & $\begin{array}{c}\text { Seguimento } \\
M(D P)\end{array}$ & $t_{\text {pré-pós }}$ & Ppré-pós & $t_{\text {pós-seg }}$ & Ppós-seg \\
\hline Competência acadêmica SSRS & $28,58(9,35)$ & $30,44(9,46)$ & $30,55(9,77)$ & $-4,1$ & $<0,0001^{* *}$ & $-0,27$ & 0,7901 \\
\hline Habilidades sociais SSRS-BR & $37,19(13,45)$ & $45,42(12,56)$ & $43,23(13,91)$ & $-10,95$ & $<0,0001^{* *}$ & 0,67 & 0,5025 \\
\hline Responsabilidade/cooop. SSRS & $19,23(7,77)$ & $22,86(6,69)$ & $21,94(7,36)$ & $-8,72$ & $<0,0001^{* *}$ & 0,96 & 0,3394 \\
\hline Asserção positiva SSRS & $10,86(4,80)$ & $13,36(4,03)$ & $12,37(4,12)$ & $-8,46$ & $<0,0001^{* *}$ & 0,31 & 0,7575 \\
\hline Autocontrole SSRS & $11,49(3,95)$ & $14,06(3,83)$ & $13,44(4,25)$ & $-10,52$ & $<0,0001^{* *}$ & 0,71 & 0,4766 \\
\hline Autodefesa SSRS & $3,28(1,84)$ & $4,51(1,53)$ & $4,19(1,59)$ & $-7,2$ & $<0,0001^{* *}$ & 0,08 & 0,9323 \\
\hline Cooperação com pares SSRS & $4,24(2,14)$ & $5,21(2,06)$ & $4,81(2,18)$ & $-7,26$ & $<0,0001^{* *}$ & 0,55 & 0,5862 \\
\hline Comptos. problema SSRS & $9,48(8,77)$ & $6,41(6,35)$ & $7,18(6,82)$ & 6,25 & $<0,0001^{* *}$ & 0,09 & 0,9297 \\
\hline Problemas externalizantes SSRS & $6,70(7,16)$ & $4,28(5,17)$ & $4,50(551)$ & 6,50 & $<0,0001^{* *}$ & 0,26 & 0,7942 \\
\hline Problemas internalizantes SSRS & $4,05(5,23)$ & $2,41(2,20)$ & $3,02(2,28)$ & 4,09 & $<0,0001^{* *}$ & $-0,24$ & 0,4307 \\
\hline
\end{tabular}

Nota: Pré-intervenção: $n=93$; pós-intervenção: $n=90$; seguimento: $n=62 .{ }^{*} p<0,05$; ${ }^{* *} p<0,01$.

ganhos segundo as professoras.

A Tabela 4 apresenta as estatísticas de GC nas escalas de competência acadêmica, habilidades sociais e problemas de comportamento, obtidas por meio do SSRS-BR, em duas avaliações. Esse grupo apresentou variação significativa apenas na subescala responsabilidade/cooperação, sinalizando ter melhora no desempe- nho na avaliação pós-espera.

Comparações entre GI e GC não acusaram diferenças em competência acadêmica. Para habilidades sociais, observou-se vantagem de GI na avaliação feita após a intervenção nesse grupo $(t=-5,63$ e $\mathrm{p}=0,002)$. A mesma tendência foi observada na escala de problemas de comportamento e nas subescalas de problemas de

Tabela 4

Estatísticas do Grupo de Comparação em Competência Acadêmica, Habilidades Sociais e Problemas de Comportamento, em Duas Avaliações

\begin{tabular}{lcccc}
\hline \multicolumn{1}{c}{ Variável } & Pré-espera $\boldsymbol{M ( D P )}$ & $\begin{array}{c}\text { Pós-espera } \\
\boldsymbol{M}(\boldsymbol{D P})\end{array}$ & $\boldsymbol{t}_{\text {pré-pós }}$ & $\boldsymbol{p}_{\text {pré-pós }}$ \\
\hline Competência acadêmica SSRS & $29,38(10,36)$ & $29,86(10,14)$ & $-1,69$ & 0,0915 \\
Habilidades sociais SSRS & $39,35(11,26)$ & $39,18(10,24)$ & $-0,61$ & 0,5398 \\
Responsabilidade/ coooperação SSRS & $21,43(6,71)$ & $22,16(6,02)$ & $-2,07$ & $0,0398^{*}$ \\
Asserção positiva SSRS & $11,06(4,33)$ & $10,74(3,22)$ & $-0,01$ & 0,9959 \\
Autocontrole SSRS & $10,99(3,05)$ & $10,88(2,87)$ & $-0,38$ & 0,7022 \\
Autodefesa SSRS & $2,80(2,84)$ & $2,75(1,49)$ & $-0,45$ & 0,6524 \\
Cooperação com pares SSRS & $4,80(2,13)$ & $5,04(2,03)$ & $-1,72$ & 0,0861 \\
Comportamentos problema SSRS & $8,09(6,11)$ & $8,49(6,12)$ & $-0,88$ & 0,3779 \\
Problemas externalizantes SSRS & $5,51(4,93)$ & $5,93(4,84)$ & $-1,41$ & 0,1605 \\
Problemas internalizantes SSRS & $3,19(2,68)$ & $3,21(2,62)$ & $-0,01$ & 0,9928 \\
\hline
\end{tabular}

Nota: $n=80 .{ }^{*} p<0,05 ;{ }^{* *} p<0,01$. 
comportamentos externalizantes e internalizantes: apenas na avaliação pós, GI apresentou menores médias de problemas de comportamento $(t=2,23, \mathrm{p}=0,02)$ e de comportamentos externalizantes $(t=1,77, \mathrm{p}=0,03)$ e internalizantes $(t=1,65, \mathrm{p}<0,0001)$.

Nas comparações referentes às subescalas de habilidades sociais, diferenças foram detectadas na pósavaliação. GI obteve melhores resultados que GC nas subescalas asserção $(\mathrm{t}=-2,34, \mathrm{p}=0,0003)$, autocontrole $(\mathrm{t}=-2,99, \mathrm{p}<0,0001)$ e autodefesa $(\mathrm{t}=$ $-1,65, \mathrm{p}<0,0001)$.

\section{DISCUSSÃO}

Este estudo, realizado com escolares entre seis e dez anos de idade, teve como objetivo avaliar a efetividade de um programa para desenvolvimento de HSPI, aplicado pelas professoras em sala de aula, no que diz respeito à melhora em HSPI, bem como a possíveis efeitos positivos nas habilidades sociais, no comportamento e no desempenho acadêmico dos alunos após a intervenção. Procurou-se também verificar a manutenção dos resultados obtidos, em avaliação de seguimento.

Em relação ao primeiro objetivo, confirmando as previsões, somente as crianças que passaram pela intervenção para o desenvolvimento de HSPI apresentaram progresso nessas habilidades. Os grupos se diferenciavam quanto às HSPI antes do início da intervenção, sendo GI superior a GC. A diferença se acentuou após a intervenção, devido à melhora no desempenho do GI, por um lado e, por outro, a perdas no GC.

Sendo as HSPI o principal foco do programa Posso Pensar, o progresso das crianças do GI nessas habilidades pode ser diretamente relacionado ao aprendizado proporcionado pela intervenção. Borges e Marturano (2002), em estudo com escolares para o desenvolvimento das HSPI, por meio de uma modalidade de intervenção primária, encontraram resultados semelhantes quanto aos ganhos obtidos.

Com relação às habilidades sociais, GI apresentou, na avaliação pós-intervenção, um aumento significativo em todas as subescalas: responsabilidade/cooperação, asserção, autocontrole, autodefesa e cooperação com os pares. GC só apresentou melhora, entre a pré e a pósespera, nas habilidades de responsabilidade/cooperação. Assim, observa-se que o GI melhora não só em relação a si mesmo, mas também quando comparado ao GC. Rodrigues et al. (2010), ao aplicarem o EPRP em crianças do primeiro ano do ensino fundamental, encontraram resultados semelhantes de aumento de habilidades sociais, autoavaliadas pelos alunos na versão do SSRS-BR para estudantes.

Ainda que o programa Posso Pensar tenha como foco principal uma classe específica de habilidades sociais, que são as HSPI, a generalização dos seus efeitos positivos para outras habilidades sociais pode ser entendida no contexto da aplicação do programa, em que a criança tem oportunidade de exercitar o reconhecimento de pistas sociais, o reconhecimento das emoções em si e nos outros, a auto-regulação emocional e a empatia. Ao passo que a empatia é em si mesma uma classe de habilidades sociais, os demais componentes trabalhados no programa são importantes blocos de construção de habilidades como o autocontrole e a própria empatia (Del Prette \& Del Prette, 2005).

Com relação aos problemas de comportamento verificados através do SSRS-BR, GI reduziu suas médias de forma significativa após o treino. GC não apresentou redução em comportamentos problemáticos entre as avaliações. Vale ressaltar que, quando comparados os grupos na avaliação pré, GI apresentava maiores dificuldades comportamentais que GC.

Evidências de atenuação dos problemas de comportamento em crianças que passam por diferentes formas de treino em habilidades sociais são recorrentes na literatura (McCabe \& Altamura, 2011). Programas com ênfase no desenvolvimento de HSPI mostram efeitos de redução da agressividade e aumento de comportamentos pró-sociais (Borges \& Marturano, 2009; 2010; Conduct Problems Prevention Research Group, 2010; Heydenberk \& Heydenberk, 2007). Em uma intervenção multimodal com características semelhantes ao Posso Pensar, no sentido de que foi conduzida pela professora e combinou o desenvolvimento das HSPI e de valores humanos no contexto escolar, Borges e Marturano (2009) obtiveram redução de conflitos e aumento de comportamentos pró-sociais em crianças de primeiro ano do ensino fundamental. Tais efeitos podem ser entendidos como influências transacionais (Burt \& Roisman, 2010), discutidas mais adiante.

No que diz respeito ao desempenho acadêmico das crianças, os grupos tinham resultados equivalentes antes da intervenção. Era esperado maior progresso no GI, tendo em vista os resultados favoráveis obtidos previamente com o EPRP, que desenvolve HSPI, sobre o desempenho escolar de crianças atendidas em uma clínica (Elias, Marturano, Motta, \& Giurlani, 2003). No entanto, era também de esperar algum progresso no GC, exposto, como o GI, ao processo de escolarização. 
Contudo, GI sinalizou progressos na avaliação pós-intervenção em todos os itens avaliados diretamente com as crianças (escrita, aritmética, leitura e escore total) e no escore de competência acadêmica avaliada pelas professoras, ao passo que GC, após a espera, somente apresentou progresso em escrita, na avaliação direta com as crianças. E mesmo nos escores de escrita a média do GI foi significativamente maior que a do GC depois da intervenção. Pode-se então supor que o progresso das crianças do $\mathrm{GC}$ refletem a exposição ao ensino regular e as diferenças entre GI e GC depois da intervenção refletem efeitos do programa sobre o desempenho.

A evidência de efeito do programa Posso Pensar sobre o desempenho em provas objetivas de escrita, leitura e aritmética parece assim demonstrada, apesar de essas habilidades não serem alvo do programa. Tais resultados são compatíveis com a recente identificação de influências transacionais entre domínios do funcionamento adaptativo (Burt \& Roisman, 2010; Grimm et al., 2010; Van Lier \& Koot, 2010). Em decorrência dos processos de retroalimentação característicos do fenômeno, intervenções para ampliar a competência das crianças em um domínio podem afetar positivamente outros domínios (Conduct Problems Prevention Research Group, 2010).

Quais mecanismos estariam na base desses efeitos no caso em exame? A melhora no desempenho pode estar relacionada às oportunidades que as crianças tiveram, durante a aplicação do Posso Pensar, para exercitar a reflexão e praticar a busca de soluções alternativas. $\mathrm{O}$ exercício da reflexão reduz a impulsividade e melhora a atenção e a persistência na tarefa, ao passo que a busca de soluções alternativas aumenta a flexibilidade cognitiva. Todos esses processos são reconhecidos na literatura como relevantes para a aprendizagem (Del Prette \& Del Prette, 2005; Matthews, Kizzie, Rowley, \& Cortina, 2010). A própria ferramenta de solução de problemas com que o programa instrumentaliza as crianças é recurso importante para o manejo dos desafios da sala de aula. Esse elenco de habilidades tem sido associado à resiliência acadêmica em crianças em risco para baixo desempenho escolar (Matthews et al., 2010).

Ainda com relação ao desempenho acadêmico vale ressaltar a concordância entre a avaliação de competência acadêmica feita pelas professoras no SSRS-BR e o escore total obtido pelos alunos no TDE, nas comparações pré x pós-intervenção. No GI, tanto a competên- cia como o desempenho melhoraram depois da intervenção, ao passo que no GC não houve mudança nas duas variáveis. Resultados semelhantes foram encontrados por Feitosa, Del Prette e Loureiro (2007), em estudo sobre a acuracidade do professor na identificação de alunos com dificuldade de aprendizagem, por meio do SSRS-BR.

Sintetizando os resultados relativos ao primeiro objetivo da pesquisa, pode-se afirmar que o programa Posso Pensar, aplicado pelas professoras na sala de aula, teve efeito positivo no funcionamento das crianças, em todas as variáveis investigadas: HSPI, outras habilidades sociais, problemas de comportamento e desempenho acadêmico. Quanto ao segundo objetivo, de verificar a manutenção dos ganhos obtidos, em avaliação de seguimento, a pesquisa forneceu resultados mistos, mas sugestivos de permanência dos efeitos do programa quatro a seis meses depois do seu encerramento. Verificou-se manutenção dos ganhos nas habilidades sociais e nos problemas de comportamento, bem como progresso nas habilidades acadêmicas básicas, entre a avaliação pós-intervenção e o seguimento.

Do ponto de vista da prevenção, a persistência dos efeitos positivos é um resultado relevante, tendo em vista que dificuldades interpessoais, comportamentais e acadêmicas nos anos escolares estão associadas ao risco aumentado de trajetórias de desenvolvimento desadaptativas (Burt \& Roisman, 2010; D'Abreu \& Marturano, 2010). Uma questão que se coloca é se tais ganhos persistiriam além do período de quatro a seis meses que mediou o término da intervenção e o seguimento. Para responder a essa questão são necessários estudos de seguimento de longo prazo. Se, por um lado, pode-se esperar que as melhoras obtidas se mantenham, devido à possibilidade de efeitos cumulativos no curso do desenvolvimento, por outro lado tem sido reiterada na literatura a importância de sessões de reforço das habilidades depois de concluída a etapa principal do programa, nos anos subsequentes (Conduct Problems Prevention Research Group, 2010).

A pesquisa tem limitações que precisam ser consideradas na apreciação do alcance de seus resultados. Quanto ao delineamento, trabalhou-se com uma amostra de conveniência, os grupos não foram randomizados e não eram estatisticamente equivalentes em termos de HSPI antes da intervenção. Quanto ao procedimento de avaliação, o fato de algumas avaliações terem sido feitas pelos próprios professores que conduziram o programa pode ter introduzido um viés positivo nos 
resultados referentes ao Grupo de Intervenção. Tal limitação parece ter sido atenuada em função dos resultados fornecidos pelos dois instrumentos aplicados diretamente com as crianças - o CHIPS e o TDE, resultados esses também indicativos de benefícios do programa.

Em contraponto às limitações, pode-se afirmar que o estudo tem algum grau de validade interna e externa. Dentro dos limites expostos, a validade interna foi assegurada pelo desenho de comparação de grupos com pré-teste e pós-teste, permitindo que as mudanças observadas nas variáveis de interesse pudessem ser atribuídas à intervenção. A validade externa foi aproximada na medida em que o programa foi conduzido em situação de vida real, no contexto de uma escola, aplicado por professores em sala de aula. Nessas condições, é plausível supor que seus resultados possam ser generalizados para situações semelhantes, de escolas de ensino fundamental (Schutz, Rivers, \& Ratusnik, 2008).

Este estudo confirma achados da literatura quanto às relações entre habilidades sociais, HSPI, desempenho acadêmico e problemas de comportamento. Os resultados acima destacados apontam para a efetividade do programa aplicado atingindo o objetivo proposto inicialmente e apontando para a importância de programas de prevenção primária para o desenvolvimento quer de HSPI ou de outras habilidades sociais como possibilidade de recursos protetores ao desenvolvimento. Vale destacar, como uma contribuição substancial do trabalho para o campo aplicado da Psicologia Escolar, a demonstração de uma parceria bem sucedida entre o psicólogo e o professor, trabalhando juntos em uma proposta de baixo custo que se mostrou promissora para a promoção do desenvolvimento das crianças no contexto da escola de ensino fundamental.

\section{REFERÊNCIAS}

Bandeira, M., Del Prette, Z. A. P., Del Prette, A., \& Magalhães, T. (2009). Validação das escalas de habilidades sociais, comportamentos problemáticos e competência acadêmica (SSRS-BR) para o ensino fundamental. Psicologia: Teoria e Pesquisa, 25, 271-282.

Barker, E. D., Vitaro, F., Lacourse, E., Fontaine, N. M. G., Carbonneau, R., \& Tremblay, R. E. (2010). Testing the developmental distinctiveness of male proactive and reactive aggression with a nested longitudinal experimental intervention. Aggressive Behavior, 36, 127-140.

Barreto, S. O., Freitas, L. C., \& Del Prette, Z. A. P. (2011). Habilidades sociais na comorbidade entre dificuldades de aprendizagem e problemas de comportamento: Uma avaliação multimodal. Psico, 42, 503-510.

Bolsoni-Silva, A., \& Del Prette, A. (2003). Problemas de comportamento: Um panorama da área. Revista Brasileira de Terapia Comportamental e Cognitiva, 5, 91-103.

Bolsoni-Silva, A., Paiva, M., \& Barbosa, C. (2009). Problemas de comportamento de crianças/adolescentes e dificuldades de pais/cuidadores: Um estudo de caracterização. Psicologia Clínica, 21, 169-184.

Borges, D. S. C., \& Marturano, E. M. (2002). Desenvolvendo habilidades de solução de problemas interpessoais no ensino fundamental. Paidéia (Ribeirão Preto), 12(24), 185193.

Borges, D., \& Marturano, E. (2009). Aprendendo a gerenciar conflitos: um programa de intervenção para a $1^{\mathrm{a}}$ série do ensino fundamental. Paideia (Ribeirão Preto), 19(42), 17-26.

Borges, D., \& Marturano, E. (2010). Melhorando a convivência em sala de aula: Responsabilidades compartilhadas. Temas em Psicologia, 18, 123-136

Burt, K. B., \& Roisman, G. I. (2010). Competence and psychopathology: Cascade effects in the NICHD Study of Early Child Care and Youth Development. Development and Psychopathology, 22, 557-567.

Caballo, V. E. (2010). Manual de avaliação e treinamento das habilidades sociais (S. M. Dolinsky, Trad.). São Paulo: Santos.

Conduct Problems Prevention Research Group. (2010). The effects of a multiyear universal social-emotional learning program: The role of student and school characteristics. Journal of Consulting and Clinical Psychology, 78, 156168.

D'Abreu, L., \& Marturano, E. (2010). Associação entre comportamentos externalizantes e baixo desempenho escolar: uma revisão de estudos prospectivos e longitudinais. Estudos de Psicologia (Natal), 15, 43-51.

De Salvo, C., Mazzarotto, I., \& Lohr, S. (2005). Promoção de habilidades sociais em pré-escolares. Revista Brasileira de Crescimento e Desenvolvimento Humano, 15(1), 46-55.

Del Prette, Z. A. P., \& Del Prette, A. (2003). Habilidades sociais e dificuldades de aprendizagem: Teoria e pesquisa sob um enfoque multimodal. In A. Del Prette \& Z. A. P. Del Prette (Eds.), Habilidades sociais, desenvolvimento e aprendizagem (pp. 167-206). Campinas: Alínea.

Del Prette, A., \& Del Prette, Z. A. P. (2005). Psicologia das habilidades sociais na infância: Teoria e prática. Petrópolis: Vozes.

Elias, L. C. S., Marturano, E. M., Motta, A. M. A., \& Giurlani, A. G. (2003). Treating boys with low school achievement and behavior problems: Comparison of two kinds of intervention. Psychological Reports, 92, 105-116.

Elias, L. C. S., Marturano, E. M., \& Motta-Oliveira, A. M. A. (2012). Eu posso resolver problemas: Um programa para o desenvolvimento de habilidades de solução de problemas interpessoais. Temas em Psicologia, 20, 521-535.

Feitosa, F. B., Del Prette, Z. A. P., Del Prette, A., \& Loureiro, S. R. (2011). Explorando relações entre o comportamento social e o desempenho acadêmico em crianças. Estudos e Pesquisa em Psicologia, 11, 442-455.

Feitosa, F. B., Del Prette, Z. A. P., \& Loureiro S. R. (2007). Acuracidade do professor na identificação de alunos com 
dificuldade de aprendizagem. Temas em Psicologia, 15, 237-247.

Gonçalves, E., \& Murta, S. (2008). Avaliação dos efeitos de uma modalidade de treinamento de habilidades sociais para crianças. Psicologia: Reflexão e Crítica, 21, 430436.

Gresham, F. M. (2004). Current status and future directions of school-based behavioral interventions. School Psychology Review, 33, 326-343.

Grimm, K. J., Steele, J. S., Mashburn, A. J., Burchinal, M., \& Pianta, R. C. (2010). Early behavioral associations of achievement trajectories. Developmental Psychology, 46, 976-983.

Heydenberk, W., \& Heydenberk, R. (2007). More than manners: Conflict resolution in primary level classrooms. Early Childhood Education Journal, 35, 119-126.

Loos, H., \& Zeller, T. J. V. (2007). Aprendendo a "brigar melhor": Administração de conflitos sem violência na escola. Interação em Psicologia, 11, 281-289.

Matthews, J. S., Kizzie, K. T., Rowley, S. J., \& Cortina, K. (2010). African Americans and boys: Understanding the literacy gap, tracing academic trajectories, and evaluating the role of learning-related skills. Journal of Educational Psychology, 02, 1757-771.

McCabe, P. C., \& Altamura, M. (2011). Empirically valid strategies to improve social and emotional competence of preschool children. Psychology in the Schools, 48, 513539.

Morgan, P. L., Farkas, G., \& Wu, Q. (2009). Kindergarten predictors of recurring externalizing and internalizing psychopathology in the third and fifth grades. Journal of Emotional and Behavioral Disorders, 17, 67-79.

Pacheco, J., Alvarenga, P., Reppold, C., Piccinini, C., \& Hutz, C. (2005). Estabilidade do comportamento anti-social na transição da infância para a adolescência: Uma perspectiva desenvolvimentista. Psicologia: Reflexão $e$ Crítica, 18, 55-61.

Rodrigues, M., Dias, J., \& Freitas, M. (2010). Resolução de problemas interpessoais: Promovendo o desenvolvimento sociocognitivo na escola. Psicologia em Estudo, 15, 831839.

Schall, R. (1991). Estimation in generalized linear models with random effects. Biometrika, 78, 719-727.
Shure, M. B. (2006). Eu posso resolver problemas: Educação infantil e ensino fundamental: Um programa de solução cognitiva para problemas interpessoais (E. M. Marturano, A. M. A. Motta, \& L. C. S. Elias, Trads.). Petrópolis: Vozes.

Shure, M. B., \& Spivack, G. (1982). Interpersonal problemsolving in young children: a cognitive approach to prevention. American Journal of Community Psychology, 10, 341-356.

Shure M. B., \& Spivack G. (1985). Children's interpersonal problem solving: Alternative solutions. Philadelphia, PA: Drexel University.

Schutz, L. E., Rivers, K. O., \& Ratusnik, D. (2008). The role of external validity in evidence-based practice for rehabilitation. Rehabilitation Psychology, 53, 294-302.

Silver, R. B., Measelle, J. R., Armstrong, J. M., \& Essex, M. J. (2010). The impact of parents, child care providers, teachers, and peers on early externalizing trajectories. Journal of School Psychology, 48, 555-583.

Stein, L. M. (1994). TDE - Teste de desempenho escolar: Manual para aplicação e interpretação. São Paulo: Casa do Psicólogo.

Sturaro, C., Van Lier, P. A. C., Cuijpers, P., \& Koot, H. M. (2011). The role of peer relationships in the development of early school-age externalizing problems. Child Development, 82, 758-765.

Van Lier, P. A. C., \& Koot, H. M. (2010). Developmental cascades of peer relations and symptoms of externalizing and internalizing problems from kindergarten to fourthgrade elementary school. Development and Psychopathology, 22, 569-582.

Witvliet, M., Van Lier, P. A. C., Cuijpers, P., \& Koot, H. M. (2009). Testing links between childhood positive peer relations and externalizing outcomes through a randomized controlled intervention study. Journal of Consulting and Clinical Psychology, 77, 905-915.

Recebido em: 28/02/2014 Primeira decisão editorial em: 05/06/2014 Aceito em: 15/07/2014 